\title{
La energía renovable en México: perspectivas desde elBalance Nacional de Energía 2012
}

\author{
Andrea Larios Vázquez* "
}

El sector energético es uno de los más importantes para la economía pues genera factores esenciales para todas las actividades productivas y bienes de consumo fundamentales para los hogares. Además, la agenda de desarrollo sustentable, ahora actualizada con la de economía verde y con la del cambio climático, sugiere la promoción de las energías renovables como una de sus estrategias cruciales. Hay dos razones principales: las fuentes fósiles aún son predominantes y generan diversos contaminantes, incluyendo los principales gases de efecto invernadero (GEIs), y dichas fuentes son no-renovables. La dependencia energética mundial sobre energías fósiles fue en 2011 de $81.7 \%$, lo que contrasta con la dependencia sobre energías renovables, que fue de $13.2 \%$. En México dichas dependencias fueron en 2012 de $92 \%$ sobre energías fósiles y de $6.8 \%$ sobre energías renovables.

Es claro, por tanto, que la economía global depende más frecuentemente de los renovables que la mexicana, y que ésta lo hace más de la energía fósil. Esta mayor dependencia, en México, es también fiscal y comercial: los ingresos petroleros representan por un lado, alrededor de $30 \%$ de los federales, y el consu- mo energético de hidrocarburos, por otro, se satisface con importaciones crecientes, lo que no necesariamente es viable en el largo plazo. Dichas dependencias tal vez ayuden a explicar la promoción diferenciada a las fuentes renovables en las administraciones recientes: mientras que en las anteriores se promocionaron ruidosamente, en la actual ya no aparece con la misma prioridad en los documentos oficiales, como en la Reforma Energética o en el Plan Nacional de Desarrollo. Con este contexto el presente artículo se motiva por analizar la participación de la energía renovable en el portafolio energético nacional con el objeto de dimensionar cuantitativamente los retos asociados al enverdecimiento de la economía. Se organiza de la siguiente manera: el primer apartado expone los conceptos energéticos que aparecen en el Balance Nacional de Energía de 2012, las contabilidades de la producción de energía primaria por tipo fuente, de la energía secundaria por tipo de producto y del consumo de energía por tipo de sector, así como la evolución de la intensidad energética. El segundo apartado expone la participación de la energía renovable en la producción primaria, donde predomina la biomasa, en la secundaria, donde está mediada por 
la producción de electricidad, y su distribución en el consumo final. Por último se ofrecen algunas conclusiones a modo de cierre.

\section{Resumen del Balance Nacional de Energía 2012}

El Balance Nacional de Energía es un documento que la Secretaría de Energía publica cada año para proveer estadísticas energéticas nacionales, y constituye una herramienta para el análisis y evaluación del desempeño sectorial. El Balance muestra los diferentes flujos de energía entre la producción y el consumo ocurridos en el transcurso de un año. De acuerdo con el Balance las fuentes de energía son aquéllas que producen energía útil directamente o por me- dio de una transformación, y se clasifican en energía primaria y energía secundaria. La energía primaria comprende los productos energéticos que se extraen o se captan de los recursos naturales. La energía secundaria, en cambio, agrupa a los derivados de las fuentes primarias que se obtienen en los centros de transformación, y que constituyen productos con características específicas para su consumo final. La Tabla 1 presenta las fuentes y productos de ambos tipos de energía. El consumo energético, por su parte, se refiere a la energía destinada a la combustión en procesos y actividades económicas, así como la que satisface las necesidades energéticas de la sociedad.

La Gráfica 1, Panel A muestra la distribución de energía primaria por

\section{Tabla I}

\section{Fuentes y productos de energía}

\begin{tabular}{l|l|l}
\hline Fuentes de energía primaria & Productos energéticos secundarios \\
\hline - Carbón mineral & - Coque de carbón \\
- Petróleo & - Coque de petróleo \\
- Gondensados & - Gas natural & - Gas licuado de petróleo \\
- Nucleoenergía & - Querosenos \\
- Hidroenergía & - Diesel \\
- Geoenergía & - Combustóleo \\
- Eólica & - Gasóleo \\
- Solar & - Productos no energéticos \\
- Bagazo de caña & - Gas seco \\
- Leña & - Etano \\
\hline Biogás & - Electricidad \\
& - Gases industriales derivados del carbón como gas \\
& & de coque y gas de alto horno.
\end{tabular}

Fuente: elaboración propia con base en Secretaria de Energía (SENER), 2012. 
tipo de fuente. La producción total en 2012 fue de 9845.91 petajoules (PJ), complementada por importaciones de 215.31 PJ y variación de inventarios de $23.07 \mathrm{pJ}$, lo que da una oferta total primaria de $10038.15 \mathrm{pJ}$. De éstos se exportan 2962.47 pJ en petróleo, carbón y condensadosy no se aprovechan 54.48 PJ, lo que resulta en una oferta bruta primaria de 7021.21 PJ. De esta oferta ocurren pérdidas primarias de 28.60 PJ, recirculación y diferencia estadística de 372.94 pJ, consumo propio primario de 270.35 PJ, transferencia interproductos de $345.64 \mathrm{pJ}$, consumo final primario de $413.61 \mathrm{PJ}$, y entrada primaria de 5590.05 PJ, que son los que se envían a los centros de transformación de energía secundaria. En cuanto a la distribución por tipo de fuente ocurre que las fuentes fósiles concentran 91.6\% (petróleo 65\%, gas natural $22.3 \%$, carbón $3.6 \%$ y condensados $1 \%)$ y que a las fuentes renovables corresponde a $6.9 \%$ (geoenergía 1.46\%, solar $0.07 \%$, eólica $0.14 \%$, hidroener- gía $1.26 \%$, biogás $0.02 \%$, leña $2.82 \%$ y bagazo de caña 1.04\%). Aunque la producción con fuente renovable es mayor a la de carbón, condensados y nuclear, la participación en la producción global es muy pequeña.

La Gráfica 1, Panel B muestra la distribución de la producción de energía secundaria por tipo de producto. La entrada primaria, identificada en el párrafo previo, se lleva a los centros de transformación donde hay pérdidas de 1813.84 PJ, resultando en una producción bruta de energía secundaria de 5525.47 PJ que, al complementarse por importaciones netas de productos secundarios, generan una oferta bruta secundaria de 7303.69 pj. En cuanto a la distribución por tipo de producto ocurre que las fuentes fósiles concentran casi 100\%: sólo 19\% de la producción de electricidad es atribuible a fuentes renovables o no fósiles, lo que resulta en que $96 \%$ de la energía secundaria es de fuente fósil (ver Gráfica 1, Panel B y Gráfica 3). 


\section{Gráfica I}

Estructura de la producción de energía primaria, y energía secundaria, 2012

- petajoules -

\section{Panel A. Energía primaria}

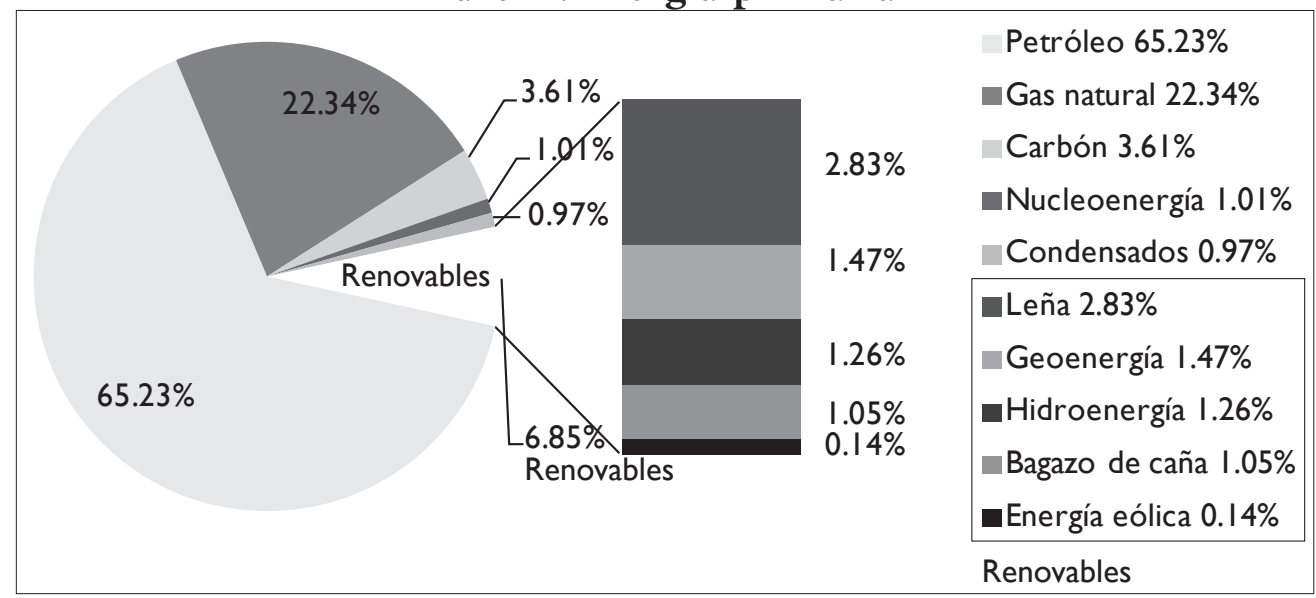

\section{Panel B. Energía secundaria}

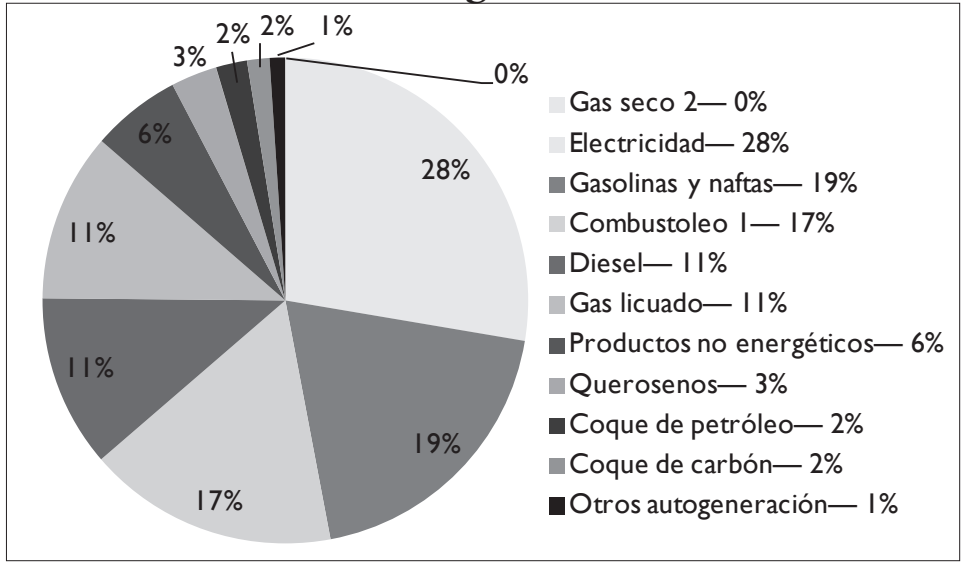

Fuente: elaboración propia con datos del Sistema de Información Energética, 2014. 
Por último, el consumo final de energía total es de 5101.84 PJ, dividido en consumo final no energético (199.87 PJ) y en consumo final energético (4 901.97 PJ), que se destinan a los diversos sectores económicos. Como se muestra en la Tabla 2, el sector transporte absorbe el mayor consumo de energía (47.8\%), dominado por consumo de gasolinas y diesel en el autotransporte. El sector industrial (31.2\%) utiliza con mayor frecuencia gas seco y electricidad impulsado por las industrias básicas del hierro y el acero, del cemento, de la petroquímica en Pemex, del vidrio y sus derivados y de la del papel, pulpa y cartón; el sector residencial (15.7\%), por su parte, consume en mayor cantidad gas licuado, leña y electricidad, mientras que el sector agrícola (3.3\%) utiliza primordialmente gas licuado, el sector comercial (2.6\%) consume principalmente petrolíferos y electricidad, y el sector público (0.6\%) consume únicamente electricidad.

Como se puede ver, los sectores económicos tienen alta dependencia a los productos fósiles (con 82\%) destacando las gasolinas, naftas, diesel y productos gasíferos, mientras que la dependencia a la electricidad es de unicamente $12.8 \%$. Sólo el sector residencial utiliza directamente de un producto renovable (leña, que representa 5\% del total). El reto de la economía verde consiste en cambiar este patrón energético con productos sustitutos que cumplan con estándares de sustentabilidad. La gráfica 2 muestra la intensidad energética, medida por kilojoules ( $\mathrm{KJ}$ ) de energía primaria consumida por unidad monetaria de producto entre 2002 y 2012. En ese período, la intensidad energética pasó de 639.51 a 664.47 PJ, lo que sugiere la ausencia de desacoplamiento entre energía consumida y valor económico. No obstante, se observan dos picos en 2005 y 2009 en los que la intensidad energética llegó a 710.75 y a 704.46 PJ, respectivamente, seguidos cada uno por un período en el que la intensidad energética disminuyó. De modo similar, en este período se incrementó la demanda energética de la población: el consumo per cápita paso de 63.32 a 75.19 gigajoules (GJ), demanda que se satisface en buena parte con un mayor volumen de importaciones de gasolinas y naftas. 


\section{Tabla 2}

Consumo de energía y tipo de energía

más utilizada por sectores, 2012

\begin{tabular}{|c|c|c|c|}
\hline Sector & $\begin{array}{c}\text { Consumo } \\
\text { total }\end{array}$ & $\begin{array}{c}\text { Tipo de } \\
\text { energía más } \\
\text { utilizada }\end{array}$ & Consumo \\
\hline \multirow{3}{*}{ Transporte } & \multirow{3}{*}{2282.4} & Gasolinas y naftas & 1506.6 \\
\hline & & Diesel & 615.2 \\
\hline & & Querosenos & 120.3 \\
\hline \multirow{2}{*}{ Autotransporte } & \multirow{2}{*}{2098.2} & Gasolinas y naftas & 1505.7 \\
\hline & & Diesel & 556.6 \\
\hline Aéreo & 121.2 & Querosenos & 120.3 \\
\hline Marítimo & 32.7 & Diesel & 32.3 \\
\hline Ferroviario & 26.4 & Diesel & 26.2 \\
\hline Eléctrico & 3.9 & Electricidad & 3.9 \\
\hline \multirow{3}{*}{ Industrial } & \multirow{3}{*}{1530.6} & Gas seco & 548.3 \\
\hline & & Electricidad & 528.1 \\
\hline & & Carbón & 109.3 \\
\hline \multirow{3}{*}{ Residencial } & \multirow{3}{*}{771.3} & Gas licuado & 287.9 \\
\hline & & Leña & 256.7 \\
\hline & & Electricidad & 189.9 \\
\hline \multirow{3}{*}{ Agrícola } & \multirow{3}{*}{160.0} & Diesel & 114.7 \\
\hline & & Electricidad & 38.9 \\
\hline & & Gas licuado & 6.4 \\
\hline \multirow{3}{*}{ Comercial } & \multirow{3}{*}{127.4} & Gas licuado & 60.0 \\
\hline & & Electricidad & 50.4 \\
\hline & & Gas seco & 10.2 \\
\hline Público & 30.2 & Electricidad & 30.2 \\
\hline Total nacional & 4901.98 & & \\
\hline
\end{tabular}

Fuente: elaboración propia con datos del Sistema de Información Energética, 2014. 


\section{Gráfica 2}

Intensidad energética, 2002-2012

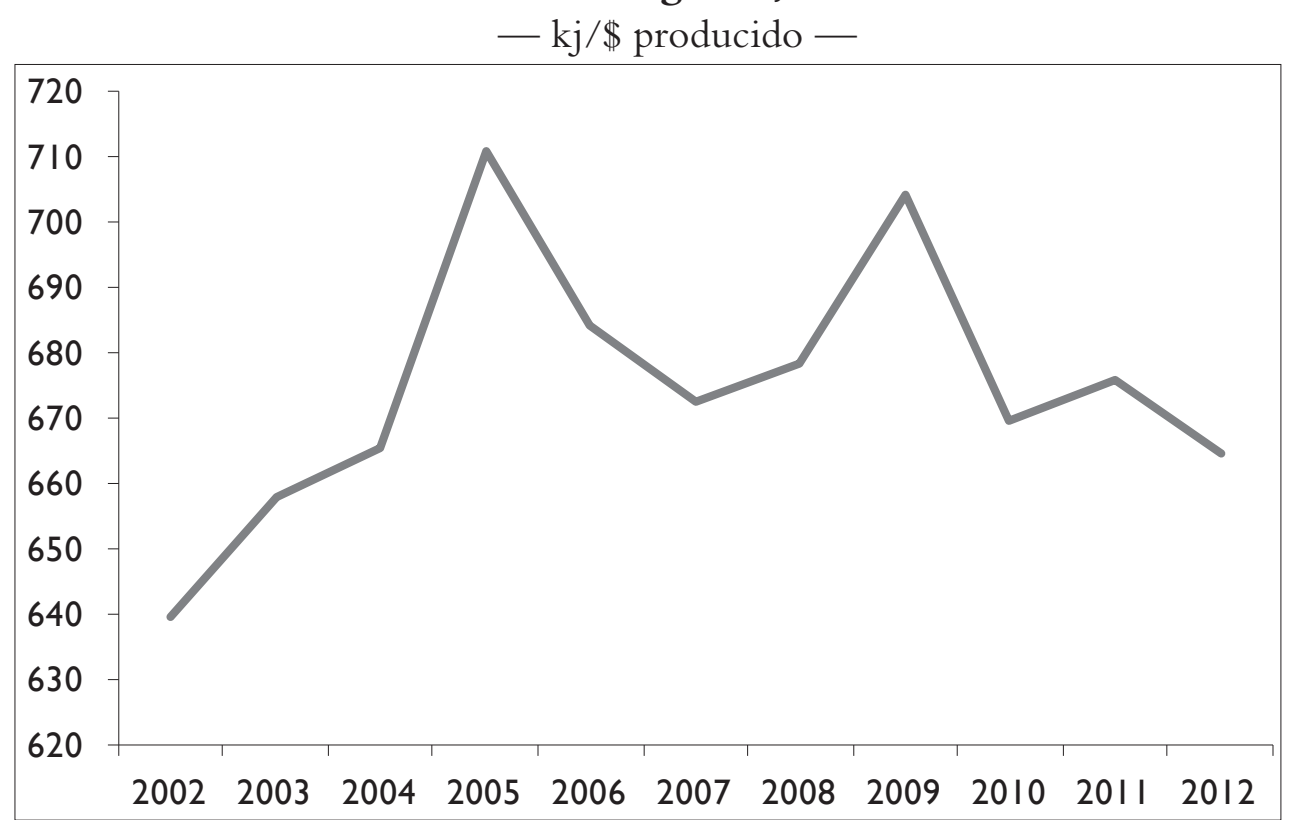

Fuente: elaboración propia con datos del Sistema de Información Energética, 2014.

\section{El papel de los renovables}

Desde la perspectiva del desarrollo sustentable hay dos problemas asociados a un patrón energético como el recién mostrado: las fuentes fósiles generan contaminantes y GEIs, y son no-renovables. Además, la creciente escasez relativa de fuentes fósiles en México ha llevado a considerar la explotación de acervos de petróleo en aguas profundas y de gas en las formaciones de lutitas, cuestiones incluidas en la reforma energética en proceso legislativo de aprobación. En contraste, la producción de energía renovable es una de las apuestas más altas para la combinación de crecimiento económico y de transición para una economía verde a nivel global: “de 2004 a 2010, la inversión en renovables presenta una tasa de crecimiento de 36\%" (PNUMA, 2011; 210). En cambio, México ha incrementado la dependencia energética sobre el carbón y sobre las importaciones de energía fósil dejando muy disminuido el papel de la energía renovable en la estrategia del crecimiento económico. Los datos del Balance indican que sólo 7\% de la energía primaria es de fuente renovable, y que esa energía se obtiene en mayor grado de la leña y del bagazo de caña, lo que muestra 
la importancia mayúscula del patrón tradicional de consumo energético en ambientes rurales en la transición hacía el desarrollo sustentable y en el manejo de los bosques. Es preocupante observar que la producción de energía solar y eólica es menor a $1 \%$ del total primario y que la hidroenergía apenas es un poco más de $1 \%$, por lo que el desarrollo de las tecnologías limpias es a todas luces insuficiente (ver Gráfica 1).

\section{Gráfica 3}

\section{Producción de energía eléctrica por tipo de fuente energética, 2012}

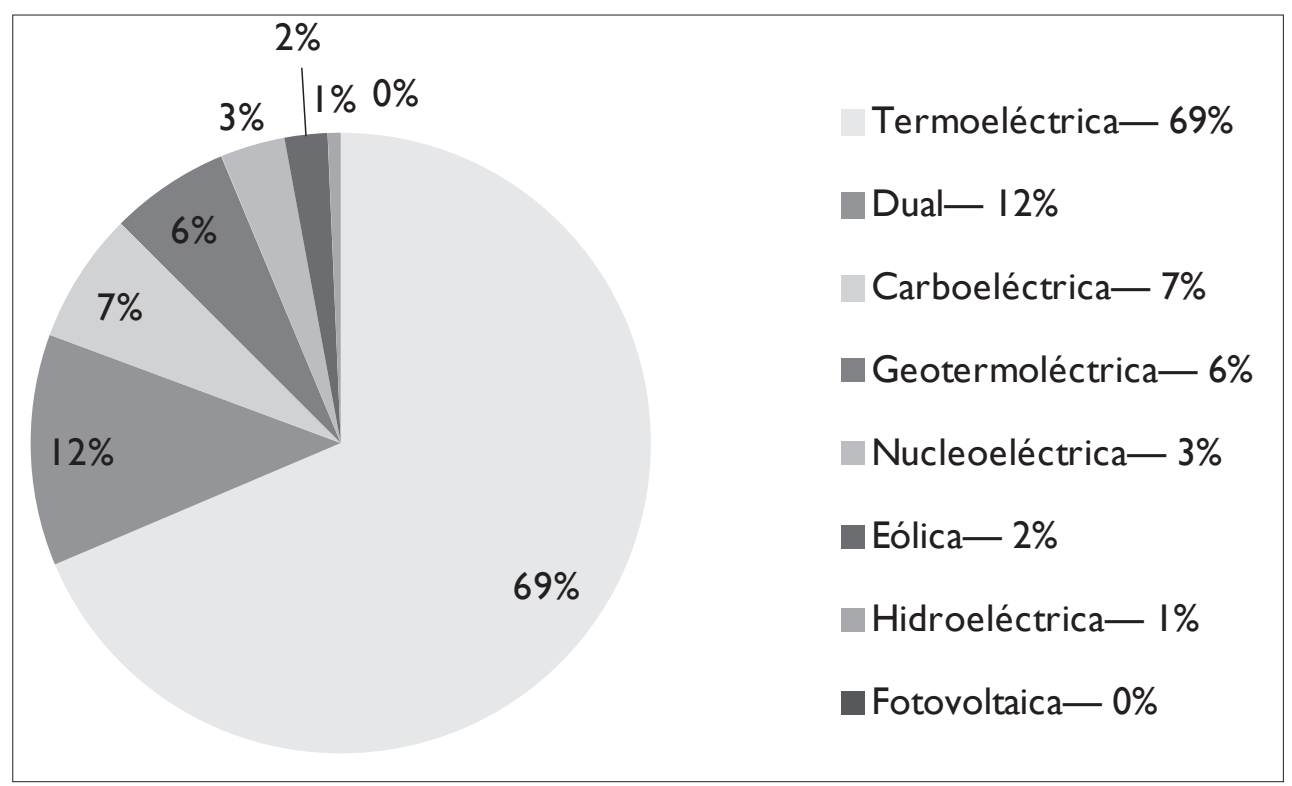

Fuente: elaboración propia con datos del Sistema de Información Energética, 2014.

Respecto a la energía secundaria, el sector eléctrico mundial le ha dado más importancia a las fuentes no fósiles: actualmente, estas fuentes suministran alrededor de una quinta parte del consumo global de electricidad. En nuestro país la producción de energía eléctrica con fuentes no fósiles representa 19\% del total, mientras que las centrales que utilizaron fuen- tes fósiles aportaron $81 \%$ restante. Por tal motivo, este sector funciona como intermediario entre energía secundaria y energía renovable, siendo la más importante la hidroeléctrica y la geotermoeléctrica, dejando atrás a la solar y a la eólica (ver Gráfica 3). El consumo final de energía también muestra una dependencia mínima a las fuentes renovables $(6.20 \%$ del total), e in- 
cluye a la energía solar, a la leña y al bagazo de caña. Estas dos últimas se conocen como biomasa, y dominan 98\% del consumo de energía renova- ble (ver Gráfica 4). Como queda claro, se consume más esta energía que la solar, la eólica y la hidroeléctrica juntas.

\section{Gráfica 4}

Consumo final de energía renovable, 2012

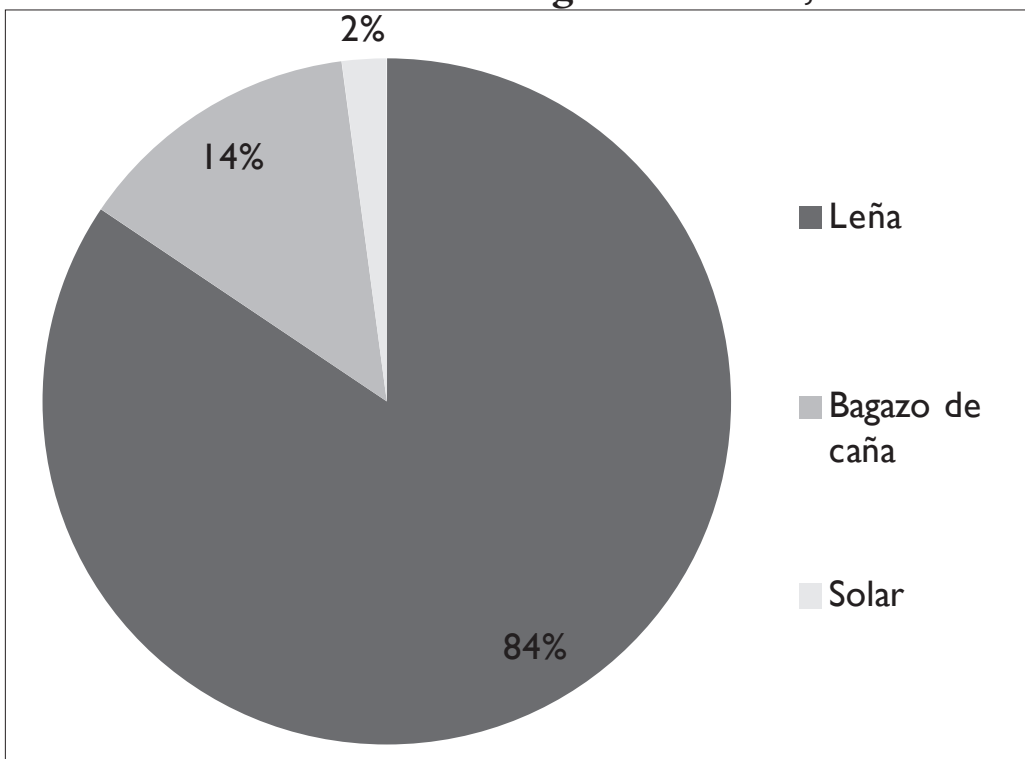

Fuente: elaboración propia con datos del Sistema de Información Energética, 2014.

\section{Conclusiones}

Los datos del Balance arrojan que México depende más de la energía fósil que el resto del mundo: 92 contra $81.7 \%$ de los totales respectivos de energía primaria, y que sigue siendo dominante la producción de petróleo y gas natural, que lo ha caracterizado como uno de los principales productores a nivel mundial. Durante los últimos años dicha producción se ha caracterizado por ser decreciente y por depender de acervos cuyo acceso es más costoso. El consumo final de energía, por su parte, está dominado por gasolinas, naftas y diesel, productos para lo que son necesarias cuantiosas importaciones. Los sectores transporte e industrial son los que más consumen energía fósil, por lo que un cambio en el patrón de consumo energético debe comenzar por ellos para obtener impactos relevantes. La producción con renovables en energía primaria es casi marginal, 
con sólo 7\%. La fuente de esta energía más importante es la biomasa, en particular la leña consumida en los hogares, lo que sugiere revisar con cierta urgencia las condiciones de su manejo, por ser un bien escaso y altamente contaminante.

Las otras fuentes renovables, incluyendo geotermia, solar, eólica, e hidroenergía, son utilizadas por el sector eléctrico para producir electricidad, que es una forma de energía secundaria. Su participación total en la producción de energía primara, no obstante, es aún muy pequeña, con apenas 3\%. Es claro que la evolución en la implementación de energía renovable en nuestro país es aún muy débil, por lo que el objetivo general de transitar al desarrollo sustentable representa retos energéticos muy profundos en un momento en el que algunas regiones del mundo ya implementan con fuerza tecnologías limpias. Estos datos sugieren que la incorporación mexicana a la energía renovable será tardía, y no está clara su promoción en el proceso corriente de reforma del sector. Quedan para un examen posterior las cuestiones asociadas a los impactos económicos de dicha incorporación, a sus efectos en la balanza comercial energética y, en general, al aprovechamiento sustentable del potencial territorial para generar energía renovable.

\section{Referencias}

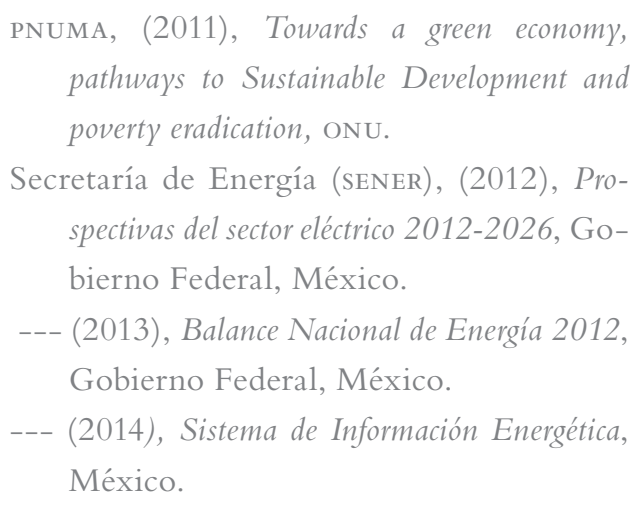

